\title{
Determination of Quality Attributes of Blue Crab (Callinectes sapidus) Meat by Electronic Nose and Draeger-Tube Analysis
}

\author{
Paul J. Sarnoski \\ Michael L. Jahncke \\ Sean F. O'Keefe \\ Parameswarakumar Mallikarjunan \\ George J. Flick, Jr.
}

\begin{abstract}
In this study, five groups of sequentially spoiled crabmeat were evaluated by a trained sensory panel, and these results were compared with the findings from a Cyranose 320 Electronic Nose and Draeger gas
\end{abstract}

Paul J. Sarnoski, Sean F. O'Keefe, and George J. Flick, Jr. are affiliated with the Department of Food Science and Technology, Virginia Polytechnic Institute and State University, Blacksburg, VA, USA.

Michael L. Jahncke is affiliated with the Department of Food Science and Technology and the Virginia Seafood Agricultural Research and Extension Center, Hampton, VA, USA.

Parameswarakumar Mallikarjunan is affiliated with the Department of Biological Systems Engineering, Virginia Polytechnic Institute and State University, Blacksburg, VA, USA.

Luis F. Martinez and Dr. Murat Balaban from the University of Florida are recognized for recommending the compressed air method. The idea of using compressed breathing air that cycles into sample containers was first developed at the University of Florida. A similar setup was used for this study. Funding for this research was provided by the Virginia Sea Grant Program, the World Food Logistics Organization, the International Association of Refrigerated Warehouses (WFLO/IARW), and the National Fisheries Institute (NFI) Scholarship Program.

Address correspondence to: Michael L. Jahncke, Department of Food Science and Technology and the Virginia Seafood Agricultural Research and Extension Center, 102 South King Street, Hampton, VA 23669 (E-mail: mjahncke@vt.edu).

Journal of Aquatic Food Product Technology, Vol. 17(3) 2008

Available online at http://jafpt.haworthpress.com

(C) 2008 by The Haworth Press. All rights reserved. 
analyzer. Using the electronic nose with filtered compressed breathing air yielded the best results. Although this approach resulted in $100 \%$ separation of the known groups, only $30 \%$ of the coded unknown samples were correctly identified. All 5 groups of samples analyzed using Draeger-Tubes were found to be significantly different at $\alpha=0.05$ using a Tukey-Kramer ANOVA statistical procedure. The coded unknown samples were correctly identified at a rate of $83 \%$. The simplicity and precision of this latter procedure may present opportunities for use of Draeger-Tubes by crab processing industries and other food processing industries as an objective method for quality control.

KEYWORDS. Electronic nose, blue crab, Draeger-tube, quality, ammonia, sensory

\section{INTRODUCTION}

The blue crab industry represents a major resource for the American seafood industry. The blue crab (Callinectes sapidus) is fished commercially from Maryland to south of Galveston, Texas; however the highest population of the species resides in the Chesapeake Bay and its tributaries. From 1968 to 2004 the annual harvest was 73 million pounds in the Chesapeake Bay alone (Bonzek et al., 2005). In recent years the harvests have dipped into the 50 million pound range. However, in 2004, the harvest was approximately 60 million pounds. The 2004 harvest translated to approximately 55 million dollars in revenue. The industry is important to the economy of the Chesapeake Bay region, and care needs to be taken to ensure that over-crabbing, habitat destruction, and other adverse environmental conditions do not lead to extinction of the blue crab.

The spoilage of crustaceans involves metabolic activities of microorganisms and enzymes. All of these processes are interrelated and occur simultaneously or at different stages of spoilage. The mechanism for the chemical breakdown of crabmeat is not as well established as for some other types of seafood, such as fish. However, it is believed the chemical breakdown is relatively similar to that of other shellfish, but not fish, as crabmeat has a higher free amino acid composition compared to fish. High levels of taurine, proline, glycine, alanine, and arginine are found in crustaceans (Martin et al., 1982). Other nitrogenous compounds, such as peptides and other nonprotein nitrogen components found in shellfish, 
form typical spoilage metabolites such as indole, ammonia, putrescine, histamine, and cadaverine (Eskin et al., 1971). An increase in tissue ammonia levels during spoilage is attributed to several enzymatic processes: deamination of free amino acids, degradation of nucleotides, and oxidation of amines (Gill, 1990). Since ammonia and other volatile amines have been implicated in seafood spoilage, it is believed that being able to measure their levels relatively quickly and easily would be of great advantage in determining quality levels.

Amines are typically produced in shellfish by the bacterial deamination or decarboxylation of free amino acids. Deamination of amino acids results in the formation of ammonia and organic acids, while decarboxylation leads to the production of amines and carbon dioxide. Breakdown of trimethylamine oxide (TMAO) may also result in the formation of trimethylamine (TMA) and dimethylamine (DMA) in certain seafood products (Martin et al., 1982). These compounds are part of a total volatile base (TVB) analysis, and methods have been derived to determine TMA and DMA concentrations separately (Jones et al., 1998; Yerlikaya and Gökoðlu, 2004). In an analysis of cod fish by Gill (1990), the relationship between TMA and subjective evaluation of odor was approximately linear.

Finding an economical way to evaluate seafood quality is difficult. Quality can be evaluated using trained certified sensory experts, but these experts are expensive to train, and periodic recertification is needed if federal and state agencies are to accept them as experts. Sensory evaluation also is expensive and difficult for the industry due to frequent personnel turnover and the extensive time commitments needed to maintain certified sensory personnel.

A possible solution to this problem is to develop a method to determine quality using electronic instrumentation. Possible instrumentation that could be used to determine quality includes the electronic nose. The electronic nose has been used in a variety of applications, including food applications (Arshak et al., 2004), and is a rapid technique with analysis time often taking less than 2 minutes.

The electronic nose uses an array of sensors designed to mimic the mammalian olfactory system's response to aromas. Instead of receptor proteins, which are part of the human olfactory system, the electronic nose in most cases uses a sensor array system. The odor molecules are drawn into the electronic nose using sampling techniques such as headspace sampling, diffusion methods, bubblers, or preconcentrators (Pearce et al., 2003). The sample odors are drawn across the sensor array, which 
induces a reversible physical or chemical change in the sensing material, in turn causing a change in electrical properties (Harsanyi, 2000). Each sensor in an array behaves in a similar manner to a mammalian protein receptor responding to different odors to varying degrees. The sensor signal is transduced into electrical signals, which are preprocessed and conditioned (usually a normalization technique is used) before being identified by a pattern recognition system.

Other methods for determining spoilage in seafood products include analyzing for ammonia and volatile amines. An AOAC-approved method for the colorimetric determination of ammonia in crabmeat exists (Steinbrecher, 1973), but this procedure is laborious and time-consuming. Ammonia can also be measured by diffusion, paper test strips, and ammonia selective electrodes, but these methods are often less sensitive compared with the colorimetric method and have interference issues, such as sodium interfering with an ammonia-sensitive electrode. The established methods require an aqueous extract. Volatile amines are also a good indicator of spoilage in seafood as total volatile base (TVB) analysis is often used to determine spoilage. In that regard, ammonia-sensitive Draeger-Tubes ${ }^{\circledR}$, used mostly for the detection of ammonia gas leaks, may work as a rapid analytical test to determine spoilage in crabmeat. The tubes have crosssensitivities with other basic substances, such as organic amines that are likewise indicated but have differing sensitivities (Dräger-Rohrchen, 2001). Since a no-specific test has previously proved somewhat effective (TVB analysis), the cross sensitivity to other amines may be advantageous for determining spoilage in shellfish.

\section{MATERIALS AND METHODS}

\section{Blue Crab Samples for Electronic Nose and Draeger-Tube Analysis}

Experiments were conducted with body meat (i.e., regular or special) obtained from a local crab processing company (Hampton, VA, USA) and harvested from the Chesapeake Bay during the same harvest season. Eight separate batches of crabmeat were spoiled (136-181 kgs/batch) and used to train the sensory panel. One of these spoilage batches was also used to train the electronic nose and one was used to establish the standard curve for the Draeger-Tube analyses. To validate the electronic nose and Draeger-Tubes, an additional batch of crabmeat was sequentially 
spoiled and used to verify the accuracy and precision of the analytical methods. The batches were separated into individual spoilage increments (18 kg/increment) and then vacuum packed in Kapak (Minneapolis, MN, USA) SealPac pouches, 0.47 liter (2.5 mil PET/LLDPE). Reference (0 day) samples were first frozen at $-18^{\circ} \mathrm{C}$ for $12-20$ hours and then stored at $-80^{\circ} \mathrm{C}$. The remaining samples were sequentially spoiled at ambient $\left(21^{\circ} \mathrm{C}\right)$ and refrigerated storage conditions $\left(4^{\circ} \mathrm{C}\right)$, frozen at $-18^{\circ} \mathrm{C}$ for $12-20$ hours, and then stored at $-80^{\circ} \mathrm{C}$ until needed for analysis. The following samples were analyzed by the electronic nose: reference ( 0 day); 6 and $9 \mathrm{~h}$ ambient; and 7, 8, and 9 day cold samples. Draeger-Tube (Dräger-Röhrchen, Luebeck, Germany) analyses were conducted on reference samples (0 day) and samples held for $4,5,6$, and 7 days at $4^{\circ} \mathrm{C}$. Samples were thawed overnight in a refrigerator $\left(4^{\circ} \mathrm{C}\right)$ before analysis.

\section{Microbial and Sensory Analysis}

Fresh-picked crabmeat was collected from the processing plant and held on ice at refrigeration temperatures $\left(<4.4^{\circ} \mathrm{C}\right)$. The initial bacterial load was enumerated by mesophilic aerobic plate count using standard AOAC-approved methods as listed in the FDA Bacteriological Analytical Manual (FDA, 2001). Twenty-five gram portions of well-mixed crabmeat were blended with $225 \mathrm{ml}$ sterile $0.1 \%$ Peptone buffer in a filter stomacher bag and stomached for $2 \mathrm{~min}$ at $230 \mathrm{RPM}$ for a 1:10 dilution. Subsequent dilutions were prepared by adding $1 \mathrm{ml}$ to $9 \mathrm{ml}$ of sterile Peptone broth. One $\mathrm{ml}$ portions were plated on $3 \mathrm{M}$ Petrifilm aerobic count plates and incubated at $35^{\circ} \mathrm{C}$ for 48 hours. Aerobic plate counts (APC) were reported as colony forming units per gram $(\mathrm{CFU} / \mathrm{g})$.

Crabmeat was divided into $5 \mathrm{lb}$. increments and spread into 10 by 16 in. lidded plastic containers for controlled decomposition at $21^{\circ} \mathrm{C}$ (ambient temperature) or $4^{\circ} \mathrm{C}$ (refrigerated storage). Crabmeat was periodically examined for sensory quality, packaged in 100-200 gram portions in Kapak 402 bags ( 1 pt. size, $61 / 2$ in. by 8 in.), vacuum sealed, labeled, and frozen overnight at $-18^{\circ} \mathrm{C}$ before placement at $-80^{\circ} \mathrm{C}$ for long-term storage. Fifty grams were used for bacteriological examination, as described above, for each spoilage increment. Reference samples were immediately bagged, frozen, and stored.

Spoiled crabmeat was evaluated for odor, taste, and texture against reference samples using a 6-member trained sensory panel. The sensory panelists underwent training for 8-12 months using the Spectrum ${ }^{\mathrm{TM}}$ Descriptive Analysis Method (Meilgaard et al., 1999) to evaluate the 
attributes of coded unknown blue crabmeat samples spoiled under refrigerated $\left(4^{\circ} \mathrm{C}\right)$ and ambient conditions $\left(21^{\circ} \mathrm{C}\right)$. A $10 \mathrm{~cm}$ line scale was used to evaluate the sensory attributes of the crabmeat samples with a score higher than $5.0 \mathrm{~cm}$ classified as a fail. Samples with scores higher than $2.5 \mathrm{~cm}$ but less than $5.0 \mathrm{~cm}$ were classified as borderline pass (mid-pass). Similarly, samples with scores lower than $7.5 \mathrm{~cm}$ but higher than $5.0 \mathrm{~cm}$ were classified as borderline fail (mid-fail). Samples were removed from $-80^{\circ} \mathrm{C}$ storage and thawed overnight in a refrigerator $\left(4^{\circ} \mathrm{C}\right)$. Packages were opened and the crabmeat was placed into 2 oz. lidded cups. The cups were heated for 20 seconds at $50 \%$ power in a 1000-watt microwave oven and immediately served to panelists. Reference samples representing different food intensity attributes were provided to the panelists prior to evaluating the crabmeat samples. Sensory and microbiological analyses were conducted at the Virginia Seafood Agricultural Research and Extension Center, Hampton, VA, USA.

\section{Electronic Nose Analysis}

Thawed 25-gram samples were placed into individual sealed 200-ml glass jars (Ball Corporation, Broomfield, CO, USA), which then were placed in a water bath at $40^{\circ} \mathrm{C}$ for 30 minutes. All samples reached equilibrium temperatures to allow headspace odors to accumulate.

\section{Manufacturer's Recommended Method}

Electronic nose analysis used individually sealed glass jars for headspace analysis. A hole was punched into the lid top to allow an entry point for the insertion of the electronic nose sniffing needle. A small piece of sticky foam (Darice Inc., Strongsville, OH, USA) was purchased from the local craft store and used as a septum. The electronic nose used for this analysis was a Cyranose $320^{\mathrm{TM}}$ (Smiths Detection, Pasadena, CA, USA), equipped with 32 polymer-conducting sensors. Ten samples of known quality crabmeat were used to build the training set for each class, and 20 separate coded crabmeat samples were analyzed to validate the system. The operating conditions used were described in the manual. The settings used were as follows: the baseline purge 10 seconds, sample draw one 25 seconds, first air intake purge 10 seconds, and second sample gas purge 75 seconds. Digital filtering and normalization were used; the substrate heater was set to $45^{\circ} \mathrm{C}$. Pump speed was set to high $(180 \mathrm{cc} / \mathrm{min})$. After analysis of each sample, a 1-min equilibration period allowed the sensors to equilibrate before being exposed to another sample. 


\section{Compressed Air Method}

Compressed tank breathing air (Airgas Mid America, Radford, VA, USA) filtered through activated carbon and moisture traps cycled through a reference and sample container was used. One of the outlets on the reference container was connected to the purge inlet of the electronic nose to ensure the nose was receiving clean, dry air that was consistent on a dayto-day basis. The flow rate through this apparatus was $15 \mathrm{ml} / \mathrm{sec}$. A tube connected the sample inlet of the electronic nose to the sample container. Ten samples were used to build the training set for each class, and 20 coded crabmeat samples were used to validate the system. Samples were not reanalyzed. The inside of the sample container was cleaned between samples. The settings used were as follows: the baseline purge 15 seconds, sample draw one 30 seconds, first sample gas purge 10 seconds, first air intake purge 10 seconds, and second sample gas purge 30 seconds. Digital filtering and normalization were used; the substrate heater was set to $45^{\circ} \mathrm{C}$. Pump speed was set to medium $(120 \mathrm{cc} / \mathrm{min})$. After each sample analysis, a 2-min equilibration period allowed the sensors to equilibrate before being exposed to another sample.

\section{Crab Reference Method}

The same settings used for the compressed air method were used for the crab reference method. The reference ( 0 day) crabmeat sample was placed in the reference container connected to the purge inlet. The flow rate through the apparatus was $15 \mathrm{ml} / \mathrm{sec}$. Ten samples were used to build the training set for each class, and 20 coded crabmeat samples were used to validate the system. Samples were not reanalyzed. Both the sample and reference containers were cleaned between samples. A 2-min equilibration period allowed the sensors to equilibrate before being exposed to another sample.

\section{Draeger-Tube Analysis}

Samples were thawed in the refrigerator $\left(4^{\circ} \mathrm{C}\right)$ overnight. Samples were brought to $40^{\circ} \mathrm{C}$ in a bag using a water bath. Bags were stomached for $1 \mathrm{~min}$ at $230 \mathrm{RPM}$. The 25 -gram samples were then transferred to a sealed glass jar. Headspace was allowed to accumulate for $10 \mathrm{~min}$. Samples were then analyzed using 2-30 ppm, or 5-70 ppm ammonia-sensitive Draeger-Tubes. The Accuro ${ }^{\circledR}$ (Dräger-Röhrchen, Luebeck, Germany) gas detector pump was used to pull $100 \mathrm{ml}$ of sample through the short-term 
detector tubes. Ten known samples were used to construct a standard curve using 2 replications for each spoilage group. Then validation of the trial was performed using unknown samples.

\section{Statistical Analysis}

Electronic nose data was processed using the PC Nose software version 6.5 (Smiths Detection, Pasadena, CA, USA), JMP IN 5.1 (SAS Institute, Cary, NC, USA), and SAS 9.1 (SAS Institute, Cary, NC, USA). The built-in PC Nose software contains a program that automatically runs canonical and principal component analysis (PCA). In addition, the raw sensor response data from the electronic nose was processed using statistical software (JMP and SAS). Canonical discriminant analysis (CDA) and stepwise discriminant analysis (SDA) were performed using SAS products. For all three methods, the following sensors were omitted from the model for stepwise discriminant analysis: 4-6, 11, 14-16, 19, 21, 22, $24,26,28,30$, and 31. Stepwise variable selection was done to eliminate sensors that showed correlation with other sensors (data) in the model. Analysis of variance (ANOVA) for the Draeger-Tube results was performed using JMP.

\section{RESULTS AND DISCUSSION}

\section{Electronic Nose Analysis}

The electronic nose has been used for food quality applications (Chantarachoti et al., 2006; Hu et al., 2005; O'Connell et al., 2001). Van Deventer and Mallikarjunan (2002) found the Cyranose 320 conducting polymer electronic nose to be superior to metal oxide and quartz crystal microbalance systems, which is why this particular electronic nose was selected for this study. For the compressed air and crab reference methods, filtered, clean, dry air from the same tank and a filtering procedure were used. The manufacturer recommends using ambient air for the test; however, it is possible that results can be affected by the varying overall quality and composition of ambient air on a daily basis. It was hypothesized that using filtered, clean, dry air rather than ambient laboratory air may provide more accurate results by eliminating sensor drift, thus achieving a more stable baseline. In addition to being able to classify differences between groups through electronic nose training procedures, validation of the model is needed in order to demonstrate the accuracy 
and precision of the electronic nose and Draeger-Tubes. This was accomplished by using known samples from multiple spoilage trials to train the electronic nose and to establish the standard curve for the Draeger-Tube analyses. The final 2 spoilage trials used coded samples to validate the electronic nose and Draeger-Tube analyses, respectively.

The compressed air method achieved $100 \%$ correct separation of all 5 groups of sequentially spoiled crabmeat as verified by a cross-validation technique (see Figure 1). This technique treats each individual sample as an unknown and then re-enters it into the statistical model. A cross-validation statistic of $100 \%$ shows each sample belongs to only one group. A poor cross validation statistic means that one or more samples can be included in multiple groups, thus decreasing the ability to distinguish between groups. For the compressed air method, all groups were significantly different at $\alpha=0.05$ using CDA and SDA. In fact, all groups were significantly different $(\mathrm{p}<0.0001)$. Separation of different known samples was excellent using the compressed air method, but identification of coded

FIGURE 1. PC nose PCA projection plot for training set data for the compressed air method.

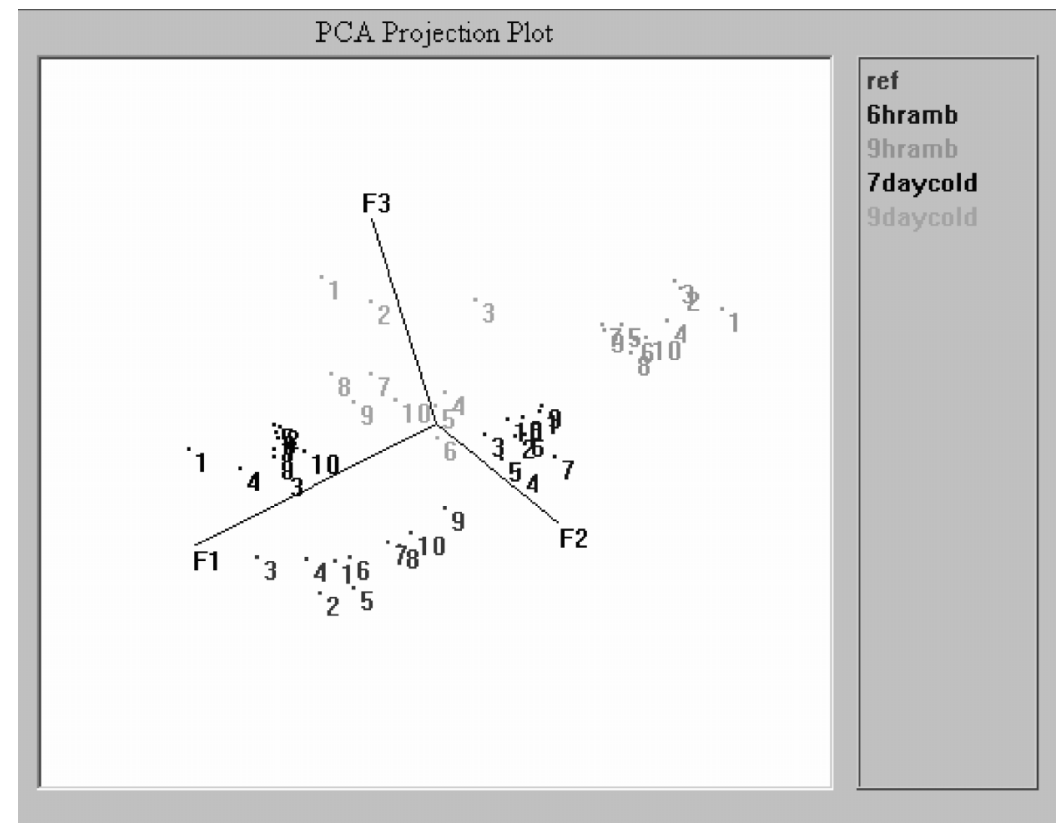


samples was not as accurate. Only $30 \%$ of the coded samples were correctly identified using CDA and SDA models. The PCA model was even less effective at correctly identifying coded samples; only $20 \%$ of the coded samples were correctly classified using this model (see Table 1).

The manufacturer's recommended method correctly separated $30 \%$ of known sample groups using PCA analysis (see Figure 2). Better separation was achieved using CDA analysis (Table 1). Correct group separation was improved to $44 \%$ using CDA analysis, and 50\% using SDA analysis, respectively. Classification of coded samples of crabmeat was low using the manufacturer's recommended method. SDA analysis yielded the best results, correctly identifying $25 \%$ of coded samples. PCA and CDA analysis correctly identified only $10 \%$ and $20 \%$, respectively, of the coded crabmeat samples.

The crab reference method used reference crabmeat (high quality meat) as a baseline for comparison of spoiled samples to unspoiled samples. Only $50 \%$ of the samples were correctly separated using PCA as the statistical method. CDA was able to correctly separate $68 \%$ of the groups, while SDA was able to correctly separate $92 \%$ of the groups at $\alpha=0.05$. CDA eliminates sensors that show correlation with other sensors from the model; in this case using this statistical method seemed to improve group separation. Correlation between electronic nose sensors was most likely high due to the samples analyzed having similar attributes. Sensory scores indicated that degrees of spoilage between samples were similar; however there is no tangible way to link the electronic nose sensor response to sensory data because the relationship of specific chemical odorants to specific sensors is proprietary. However, the crab reference method should

TABLE 1. Comparative results for electronic nose studies

\begin{tabular}{lccc}
\hline Setup & $\begin{array}{c}\text { Statistical } \\
\text { Method }\end{array}$ & $\begin{array}{c}\text { Group } \\
\text { Separation (\%) }\end{array}$ & $\begin{array}{c}\text { Correct } \\
\text { Identification (\%) }\end{array}$ \\
\hline Manufacturer's & PCA & 30 & 10 \\
Recommended & CDA & 44 & 20 \\
Compressed Air & SDA & 50 & 25 \\
& PCA & 100 & 20 \\
Crab Reference & CDA & 100 & 30 \\
& SDA & 100 & 30 \\
& PCA & 50 & 20 \\
& CDA & 68 & 35 \\
\hline
\end{tabular}


FIGURE 2. PC nose PCA projection plot for training set data for the manufacturer's recommended method.

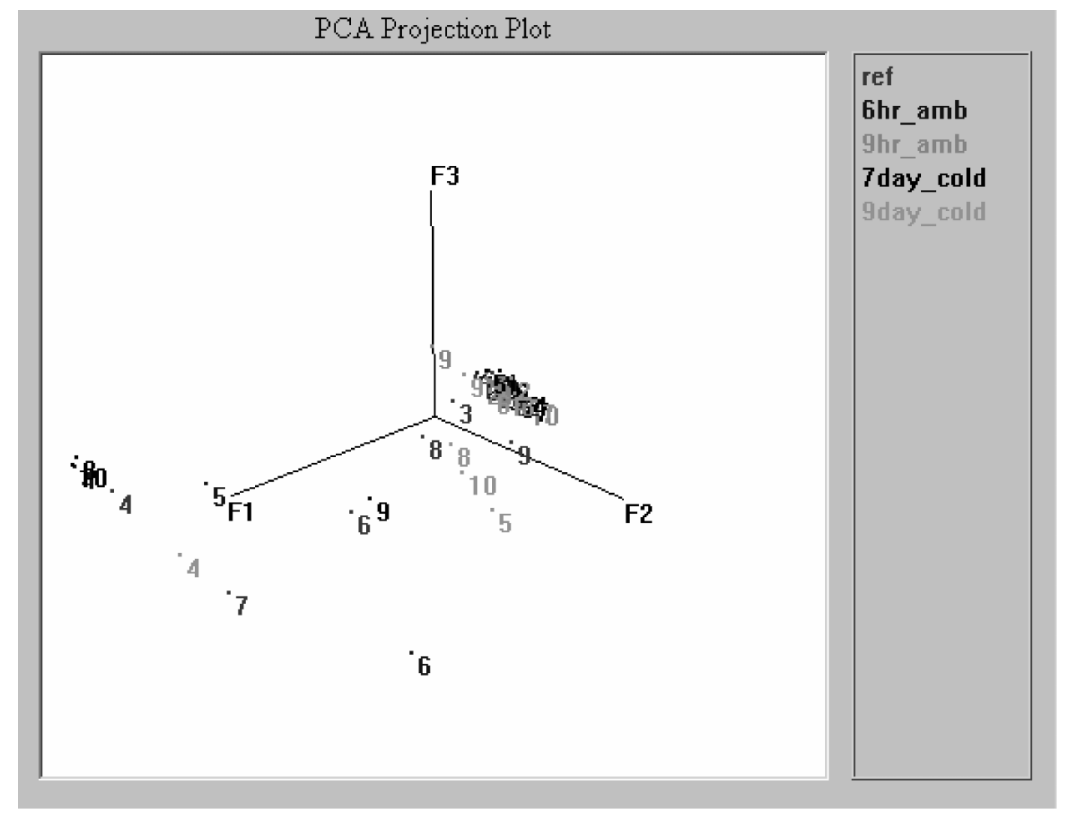

have accentuated differences due to spoilage, since spoilage attributes should have been the only differences between the samples. For the coded crabmeat samples, CDA classified $35 \%$ of samples correctly. The PCA and SDA results were less accurate, correctly identifying only $20 \%$ of the coded samples. It was expected that SDA analysis on the coded crabmeat samples would result in the highest correct classification method, since for the previous analyses, separation and identification of unknown samples were highest using this statistical method.

The compressed air method had the highest success rate in separating and identifying the coded samples. The manufacturer's recommended analysis procedure produced the poorest results for separation and identification of the coded crabmeat samples. These results suggest that methods using clean dry tank air will produce better group separation. However, low rates of correct classification of coded samples were also obtained using clean, dry, filtered air.

ANOVA analysis and declassification of some of the groups were also conducted. ANOVA analysis of individual sensors in the 32-sensor array was conducted to see if one sensor could be used to determine spoilage 
levels. In these analyses, one sensor was not more effective than PCA, CDA, or SDA for correctly identifying spoilage levels. Another technique employed was to group together the 6 and $9 \mathrm{~h}$ ambient samples, the 7 and 9 day cold samples, and reference samples ( 0 day) forming a 3-group model. Sensory evaluations indicate that the ambient increment and cold spoiled increment had similar attributes regardless of spoilage time (see Table 2). However, combining the groups did not considerably improve the accuracy of the unknown identification results. Water may have interfered with the sensors, confounding the results. When the water-sensitive sensors, numbers 5, 6, 23, and 31 according to The Practical Guide to the Cyranose 320 ${ }^{\mathrm{TM}}$ (Cyrano Sciences, 2001), were omitted from the model, group separation was still poor. Perhaps these sensors are also sensitive to the polar components present in the aroma profile of spoiling crabmeat.

In order to gain insight into why the coded sample results differed from the training data using known samples, the coded sample results were plotted using the training data of the compressed air method (see Figure $3)$. Most of the coded samples were identified as low quality even though high quality ( 0 day) samples were present in the coded sample set. None of the 0 day samples were correctly identified. There may have been a "hold over" affect from the more spoiled samples that affected the results of the less spoiled samples, even though the sample containers were cleaned after each trial and the sensors were purged according to the prescribed settings of the Cyranose 320 manual. Since samples were of similar quality (according to microbial and sensory analysis) for both known and unknown samples, differences between the training data and the coded sample data may be due to deficiencies in the sensor purging system of the electronic nose.

The electronic nose may not have been sufficiently sensitive to distinguish between minor differences between classes of spoiled crabmeat.

TABLE 2. Aerobic plate counts and sensory results for samples used for the Cyranose $320^{\mathrm{TM}}$ analysis

\begin{tabular}{lcl}
\hline Sample & APC $($ CFU/g) & Sensory Classification \\
\hline Reference (0 day) & $9.2 \cdot 10^{4}$ & High pass \\
6 hr. ambient & $4.6 \cdot 10^{5}$ & Mid-pass \\
9 hr. ambient & $1.0 \cdot 10^{6}$ & Mid-pass/low pass \\
7 day cold & $7.5 \cdot 10^{6}$ & Low fail/mid-fail \\
9 day cold & $4.0 \cdot 10^{7}$ & Mid-fail/high fail \\
\hline
\end{tabular}


FIGURE 3. Compressed air method CDA plot with coded unknown samples plotted.

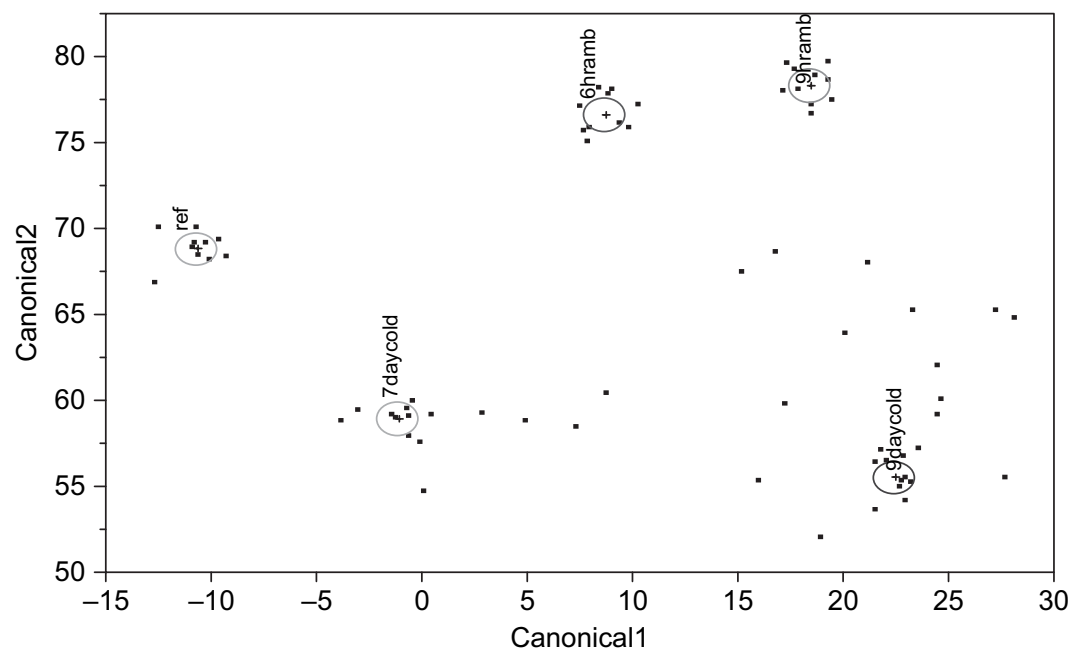

The crabmeat samples were of varying degrees of spoilage, which to the electronic nose may not have been perceived as a noticeable difference. Ammonia, which is associated with crabmeat spoilage, is soluble in water. This may have led to ammonia not being at a detectable level by the electronic nose, since crabmeat also contains an appreciable amount of water. In addition, no recommended calibration procedure exists for this electronic nose. It is also possible that as the conducting polymer sensors age, sensor drift may be an issue with electronic nose devices (Hines et al., 1999). These issues have been shown to occur when the electronic nose has been used to quantitatively identify components (Harper, 2001). This study is similar to a quantification study, since as spoilage increases, the intensity of spoilage attributes (odors) increase.

Suggestions for improving the electronic nose results include using fewer sample groups, bootstrapping, and neural networks. Instead of using 5 groups for training, use a high quality group and a low quality group. Bootstrapping is an approach best utilized for validating a given model, especially when a small sample size is used. Bootstrapping creates a larger sample size by using real data to create imaginary data. For example, 30 real observations can create 1000 "bootstrap" observations. This approach is best when the error in analysis is attributed to small sample 
size. The bootstrap method was used in a study of beef spoilage, and it improved the classification accuracy from $87 \%$ to $98 \%$ (Balasubramanian et al., 2004).

Artificial neural networks are mathematical creations inspired by the biological nervous system. An artificial neural network consists of a lattice of information processing elements called neurons, which are connected together in a certain way (known as the architecture). The strengths of these connections are called synaptic weights. These weights are determined either during a training (or learning) phase for supervised neural networks or by an algorithm for unsupervised neural networks (Gardner and Bartlett, 1999). Therefore, a neural network can "learn" as data is imputed in, whereas a pattern recognition technique simply looks for patterns. The use of neural networks significantly improved pattern recognition for electronic nose systems (Zhang et al., 2003) and was found to be effective for quantifying different spice mixture compositions (Zhang et al., 2005). Due to time constraints, this technique was not applied to this data set.

\section{Draeger-Tube Analysis}

The data from the sequentially spoiled samples was plotted in a standard curve, using an average of 2 replications for each group (see Figure 4). These data show an expected pattern: as the product spoils, the ppm value for ammonia increases. These data fit an exponential equation rather than a linear equation. This is not surprising since microbial growth follows an exponential equation (Marr, 2000). ANOVA analysis was conducted on the Draeger-Tube results (see Figure 5). Means were separated using a Tukey-Kramer statistical test.

Coded samples were analyzed using the Draeger-Tube method (See Table 3). Correct classification of $83 \%$ of the coded samples was achieved. Code 411 was the only sample totally misclassified-the sample was a 7-day cold sample, but was classified as a 6-day cold sample by ANOVA and the standard curve. Codes 247 and 302 were not significantly different from the 0-day cold or 4-day cold samples according to Tukey-Kramer ANOVA analysis (See Figure 6). These two samples were classified into either group. These results are not surprising, since the sensory analysis and aerobic plate counts showed that the 0-day cold and 4-day cold samples were quite similar (see Table 4). Sensory analysis classified the 0-day cold and 4-day cold samples as having attributes associated with a mid quality sample. When spoilage was estimated using the 
FIGURE 4. Ammonia standard curve. Both linear and exponential equations are plotted on the same graph. The exponential equation better fits the data as is reflected in the $R^{2}$ value.

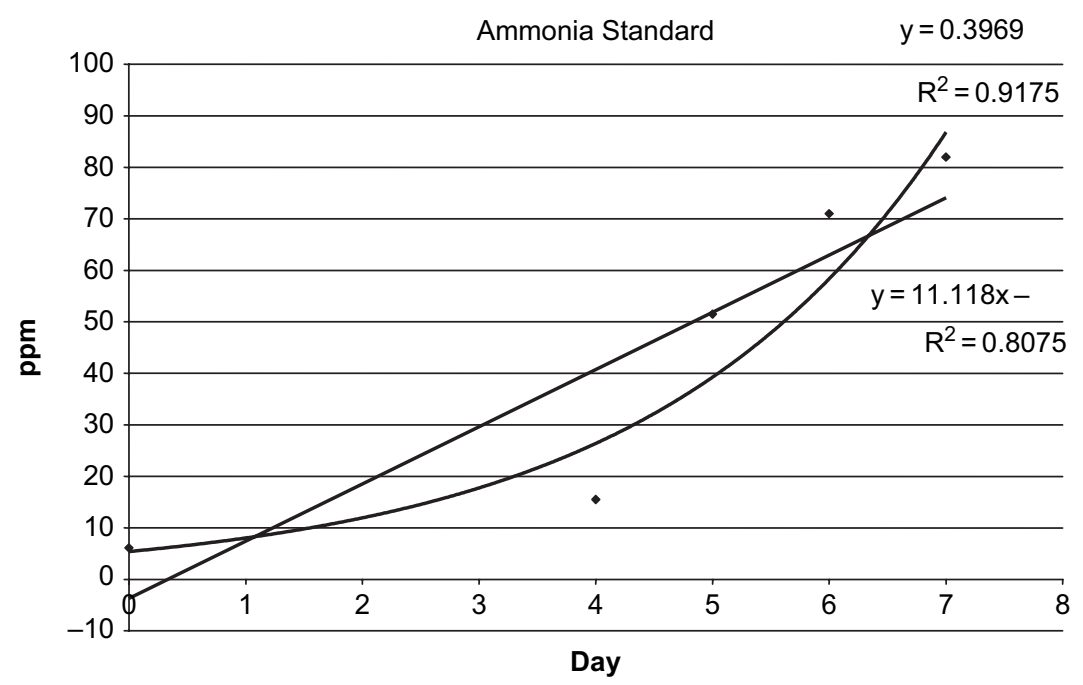

FIGURE 5. Ammonia data analyzed by the Tukey-Kramer test for One-Way ANOVA. All groups were found to be significantly different at the $\alpha=0.05$ level.

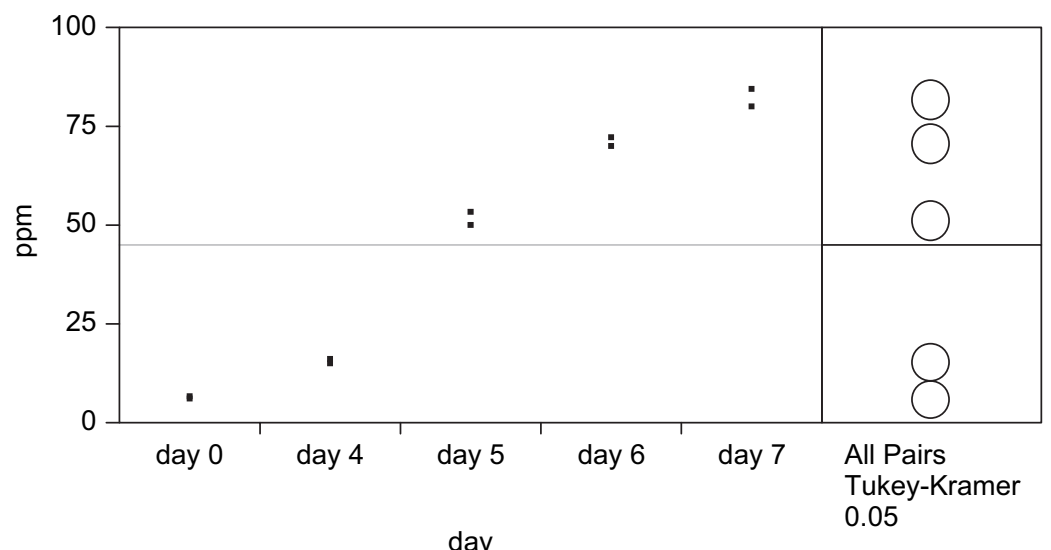


TABLE 3. Draeger coded unknown results

\begin{tabular}{lcccc}
\hline Code & $\begin{array}{c}\text { Parts Per } \\
\text { Million (ppm) }\end{array}$ & $\begin{array}{c}\text { Estimated Day } \\
\text { (Standard Curve) }\end{array}$ & Actual Day & $\begin{array}{c}\text { ANOVA } \\
\text { Classification (Day) }\end{array}$ \\
\hline 411 & 71 & 6.4 & 7 & 6 \\
949 & 55 & 6.0 & 5 & 5 \\
247 & 8 & 3.1 & 0 & $0 / 4$ \\
455 & 85 & 6.6 & 7 & 7 \\
339 & 41 & 5.6 & 5 & 5 \\
302 & 10 & 3.4 & 4 & $0 / 4$ \\
\hline
\end{tabular}

Parts per million (ppm) values were determined by Draeger-Tubes analysis and standard curve (exponential) was used to compute the estimated day. JMP IN was used for the ANOVA classification

FIGURE 6. Ammonia data analyzed by the Tukey-Kramer test for One-Way ANOVA with coded unknowns. A means comparison analysis was performed on this data and the statistical procedure identified into which spoilage day each coded unknown should be classified.

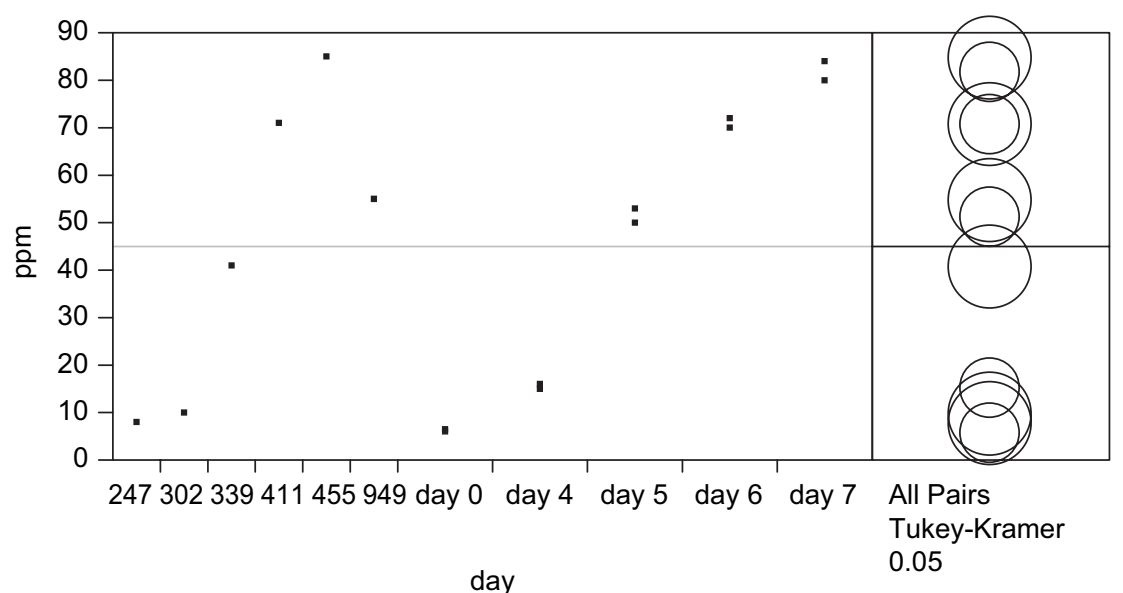

standard curve, code 247 was estimated to be a 3.1-day sample (closer to 0 day), and code 302 was estimated to be a 3.4-day sample (closer to a 4 day), thus corresponding closely with the actual spoilage increment. Most importantly, both samples were classified correctly as "pass." What is important about this method is the sensitivity of the Draeger-Tube 
TABLE 4. Aerobic plate counts (APC) and sensory results of samples used for Draeger-Tubes analysis

\begin{tabular}{lcl}
\hline Sample & APC $($ CFU/g) & $\begin{array}{c}\text { Sensory } \\
\text { Classification }\end{array}$ \\
\hline Reference (0 day) & $1.65 \cdot 10^{5}$ & Mid pass \\
4 day cold & $8.5 \cdot 10^{5}$ & Mid Pass \\
5 day cold & $1.6 \cdot 10^{6}$ & Low pass \\
6 day cold & $3.2 \cdot 10^{6}$ & Low pass/low fail \\
7 day cold & $1 \cdot 10^{7}$ & Mid fail/high fail \\
\hline
\end{tabular}

method compared to the electronic nose method. This method may be sufficiently sensitive (at least for crabmeat) to separate crabmeat quality into different categories. Thus, the coded samples 247 and 302 were considered to be correctly classified.

The Draeger-Tubes rely on a simple chemical reaction. The tubes contain a $\mathrm{pH}$ indicator. The reaction principle is that as $\mathrm{NH}_{3}$ (a base) is exposed to the $\mathrm{pH}$ indicator, a color change occurs due to a rise in $\mathrm{pH}$, demonstrated by a change in color from yellow to blue inside the tube. Perhaps this reaction can still be used, but it may need to be modified to develop a dipstick test that industry could easily use. The Draeger method is simple and more rapid compared with the chemical determination of ammonia and total volatile bases. The technology may also be easily transferable to industry because many seafood processors already use Draeger-Tubes to check for ammonia refrigerant leaks.

\section{Comparison of Draeger-Tube and Electronic Nose Methods}

It is important to note that the training samples and the samples used for validation (coded unknown samples) were from 2 different batches. The sample groups $(0,4,7$ day cold, etc.) for training and validation were of the same or similar spoilage time, but they were from different crabmeat batches. Duplicate samples were run during training and validation and yielded different results using the electronic nose (refer to Figure 2). Draeger-Tube and sensory analysis were able to recognize similar spoilage groups across different batches of crabmeat; however the electronic nose often misclassified or even classified replicate samples into different spoilage groups. This suggests that the Draeger-Tube procedure was more robust than the electronic nose under conditions of use in this study. 


\section{CONCLUSIONS}

The results for the coded validation samples indicate that if the Cyranose 320 electronic nose is used as a quality indicator for crabmeat, improvement in its technology is needed. The results indicate that the DraegerTubes show promise for determining spoilage levels for crabmeat and could be used as a quality control procedure by industry. More research is needed to determine if the method works across many batches, seasons, environments, and species.

\section{REFERENCES}

Arshak, K., Moore, E., Lyons, G. M., Harris, J., and Clifford, S. 2004. A review of gas sensors employed in electronic nose applications. Sensor Rev. 24: 183-195.

Balasubramanian, S., Panigrahi, S., Logue, C. M. Marchello, M., Doetkott, C., Gu, H., Sherwood, J., and Nolan, L. 2004. Spoilage identification of beef using an electronic nose system. Trans. ASAE. 47: 1625-1633.

Bonzek, C., Fegley, L., Hoenig, J., Miller, T., O’Reilly, R., Orner, D., Sharov, A., Terceiro, M., and Vaughan, D. 2005. 2005 Chesapeake Bay Blue Crab Advisory Report. Chesapeake Bay Stock Assessment Committee.

Chantarachoti, J., Oliveria, A. C. M., Himelbloom, B. H., Crapo, C. A., and McLachlan, D. G. 2006. Portable electronic nose for detection of spoiling Alaska pink salmon (Onchorhynchus gorbuscha). J. Food Sci. 71: S414-S421.

Cyrano Sciences. 2001. The Practical Guide to the Cyranose 320. Rev. C. Pasadena, CA: Cyrano Sciences, Inc.

Dräger-Rohrchen. 2001. Instructions for Use. 9th ed. Luebeck, Germany: Dräger.

Eskin, N. A., Henderson, H. M., and Townsend, R. J. 1971. Biochemistry of foods. New York: Academic Press, Inc.

FDA. 2001. Bacteriological Analytical Manual Online. 8th ed.: U.S. Food and Drug Administration.

Gardner, J., and Bartlett, P. 1999. Electronic Noses Principles and Applications. New York: Oxford University Press.

Gill, T. 1990. Objective analysis of seafood quality. Food Rev. Int. 6: 687-697.

Harper, W. J. 2001. The strengths and weaknesses of the electronic nose. Adv. Exp. Med. Biol. 488: 59-71.

Harsanyi, G. 2000. Polymer films in sensor applications a review of present uses and future possibilities. Sensor Rev. 20: 71-81.

Hines, E. L., Llobet, E., and Gardner, J. W. 1999. Electronic noses: a review of signal processing techniques. Circuits, Devices and Systems, IEE Proceedings 146: 297-310.

Hu, X., Quillin, R. C., Matanin, B. M., Cheng, B., Mallikarjunan, P., and Vaughan, D. 2005. Development of non-destructive methods to evaluate oyster quality by electronic nose technology. ASAE Paper No. 056097. St. Joseph, MI: ASAE. 
Jones, P. R. H., Ewen, R. J., and Ratcliffe, N. M. 1998. Simple methods for the extraction and identification of amine malodurs from spoiled foodstuffs. J. Food Compos. Anal. 11: 274-279.

Marr, A. G. 2000. Growth kinetics, bacterial. Lederberg, J., ed. In: Encyclopedia of Microbiology. 2nd ed. San Diego: Academic Press. pp. 584-589.

Martin, R., Flick, G., Hebard, C., and Ward, D. 1982. Chemistry and Biochemistry of Marine Food Products. Westport, CT: AVI Publishing Co. pp. 137-368

Meilgaard, M., Civille, G. V., and Carr, B. T. 1999. In: Sensory Evaluation Techniques. 3rd ed. Boca Raton, FL: CRC Press. pp. 168-172.

O'Connell, M., Valdora, G., Peltzer, G., and Martin Negri, R. 2001. A practical approach for fish freshness determinations using a portable electronic nose. Sens. Actuators B 80: 149-154.

Pearce, T. C., Schiffman, S. S., Nagle, H. T., and Gardner, J. W. 2003. Handbook of Machine Olfaction. Weinheim: Wiley-VCH.

Steinbrecher, K. 1973. Collaborative study of the determination of ammonia as an index of decomposition in crabmeat. J. AOAC Int. 56: 598-599.

Van Deventer, D., and Mallikarjunan, P. 2002. Comparative performance analysis of three electronic nose systems using different sensor technologies in odor analysis of retained solvents on printed packaging. J. Food Sci. 67: 3170-3183.

Yerlikaya, P. and Gökoðlu, N. 2004. Quality changes of blue crab (Callinectes sapidus) meat during frozen storage. J. Food Qual. 27: 83-89.

Zhang, H., Balaban, M. Ö., and Principe, J. C. 2003. Improving pattern recognition of electronic nose data with time-delay neural networks. Sens. Actuators B 96: 385-389.

Zhang, H., Balaban, M. Ö., Principe, J. C., and Portier, K. 2005. Quantification of spice mixture compositions by electronic nose: Part 1 . Experimental design and data analysis using neural networks. J. Food Sci. 70: E253-258. 